\title{
Exact inflationary solutions
}

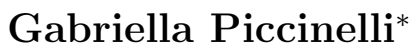 \\ Instituto de Astronomía \\ Universidad Nacional Autónoma de México \\ Apartado Postal 70-264, 04510, México D. F., México. \\ Tonatiuh Matosin, \\ Instituto de Física y Matemáticas \\ Universidad Michoacana de San Nicolás de Hidalgo \\ Apartado Postal 2-82, Morelia 58040, Michoacán, México. \\ and \\ Merced Montesinos \\ Departamento de Física \\ Centro de Investigación y de Estudios Avanzados del IPN \\ Apartado Postal 14-740, 07000, México D.F., México.
}

We present a new class of exact inflationary solutions for the evolution of a universe with spatial curvature, filled with a perfect fluid, a scalar field with potential $V_{ \pm}(\phi)=\lambda\left(\phi^{2} \pm \delta^{2}\right)^{2}$ and a cosmological constant $\Lambda$. With the $V_{+}(\phi)$ potential and a negative cosmological constant, the scale factor experiments a graceful exit.

We give a brief discussion about the physical meaning of the solutions.

PACS: 98.80.Dr 12.10.Gq

\section{INTRODUCTION}

Inflation is a useful concept for cosmology, both as a theory of initial conditions and providing the physical conditions for seeding the universe large scale structure (see e.g. Linde [1]). After the old-inflationary model [2], where inflation ended through a firstorder phase transition in which the true-vacuum bubbles never percolated, inflation has been traditionally achieved for a slow rollover scalar potential $V(\phi)$, assuming it is the

\footnotetext{
*e-mail: gabriela@astroscu.unam.mx

${ }^{\dagger}$ Permanent Address: Departamento de Física, CINVESTAV-IPN, Apartado Postal 14-740, 07000, México D.F., México.

e-mail: tmatos@fis.cinvestav.mx

${ }^{\ddagger}$ Current Address: Department of Physics and Astronomy, University of Pittsburgh, Pittsburgh, PA 15260, USA.

e-mail: merced@fis.cinvestav.mx
} 
dominant term in the total energy density and neglecting the contributions of the kinetic energy $\frac{1}{2} \dot{\phi}^{2}$, in order to have a de Sitter-like inflation, with a (quasi) exponential solution. Subsequently, recognizing that an inflationary expansion can be defined just by $\ddot{a}>0$ (where $a$ is the universe scale factor), models with a steeper potential have been considered, leading to power-law inflation (see e.g. [3]). These models can be related, by a conformal transformation, to a scalar-tensor gravity theory with a standard inflationary potential, thus introducing modifications in the gravitational sector too [4.

Many inflationary models have been proposed up to now (for a review see e.g. [5]), all of them with a more or less pronounced fine-tuning problem. Recently, in order to keep pace with astrophysical observations that seem to point to low $-\Omega$ values ( $\Omega$ is the universe density parameter), a double-round inflation model that leads to $\Omega<1$ has been extensively developed (see [6] and references there in).

As we mentioned, in the usual approach to the early universe evolution, only the conjectured dominating energy density during each epoch is kept as the source for the universe expansion, neglecting all the sub-dominant components. In this way, the inflationary solutions use to emerge from many assumptions that considerably simplify the equations. A work from Chimento and Jakubi [7] analyses some exact solutions in a many-components cosmology, showing the limitations imposed by the slow-rollover approximation. They find that the fluid source and/or the spatial curvature may dominate the early universe evolution and inhibit inflation. Schunck and Mielke [8.9] present a method for finding exact solutions using the Hubble parameter as a time coordinate; they find some exact inflationary solutions and compare them with COBE results in some approximation.

Another possibility for the cosmological scenario is the existence of a cosmological constant term. It has been widely exploited in structure formation models and it has been mainly considered in order to extend the Universe lifetime [10]. This term is expected to dominate at later epochs but it is anyhow a possible component of the very early Universe.

An important issue in constructing a successful inflationary model is to find a fundamental theory that naturally contains it. An appealing framework for inflationary fields is higher-dimensional theories. There, like in Kaluza-Klein (KK) and in superstring theories (SS), the scalar field -the dilaton- is a natural component of the field equations. Nevertheless, inflationary models from higher-dimensional theories have encountered serious problems : either they do not inflate enough (see [11] and references therein) or they have a 
graceful exit problem, as in [12]. In [13] the fermionic part, together with a Higgs potential, have been added to an eight-dimensional theory in order to recover the standard model from a KK theory. Thus, KK becomes an n-dimensional model for the standard model, coupled to gravity. If we introduce the FRW-metric in a higher-dimensional KK theory, the fermionic sector vanishes (gauge fields define a privileged direction) and the scalar sector reduces to one scalar field [14].

On another hand, a general feature of superstring cosmology is that until the dilaton field settles near a minimum of its potential, its kinetic energy dominates the potential energy, preventing inflation. In this context, the best possibility should be that the dilaton settles down and inflation is driven by other fields. Nevertheless, for $\phi$ to be trapped in a minimum of its potential, some very strict constraints on the initial conditions have to be satisfied. Moreover, the dilaton potential typically has minima with negative vacuum energy density and this carries many problems for cosmology: the difficulties to avoid a minimum with negative cosmological constant and an unacceptably high variation of the gravitational coupling (see e.g. [15]).

Successful inflation may occur due to chiral fields, under certain conditions, once the dilaton and moduli fields are stabilized by demanding an S-duality [16] invariant potential [17]. Another viable model, called false vacuum inflation [18], have been built in a class of supergravity models that follow from orbifold compactification of superstrings, albeit again with significantly constrained parameters. It requires two scalar fields -one rolling and the other trapped in a false vacuum state- and the false vacuum energy can give the dilaton a suitable minumum during inflation.

Another interesting possibility arises when the duality symmetry is invoked. Gasperini and Veneziano [19] use the scale factor duality (SFD) and the time inversion of the string effective equations to deduce the existence of a scenario named "pre-big-bang" which is associated, by SFD and time inversion, to the "post-big-bang" epoch, namely, the actual epoch. In this context, the big bang epoch means the transition between these two dual phases. In [20], starting from the multidimensional Brans-Dicke theory with a perfect fluid, solutions to the equations of motion were found which contain a singularity in the curvature. This approach also admits solutions without singularities when a self-dual potential is introduced for the dilaton field. The scale factor grows non-monotonically through three phases of accelerated expansion, contraction, expansion, before the final decelerated expan- 
sion (standard cosmological model). Inflation can be accomodated in these three phases of accelerated evolution.

Keeping in mind all these possibilities for the universe content and features -dimensions, curvature, different inflationary fields and episodes-, in this work we kept all the terms

possibly envolved in the early universe dynamics and we were able to find exact inflationary solutions for a closed universe and different signs of the cosmological constant. In each case, $\Lambda$ and $\rho_{0}$ will be defined by the parameters of the theory. The requirement that $\rho>0$ will put constraints on the allowed values of the parameters and hence on the form of the solutions.

In section II we briefly present the model and in the two following sections we give two classes of inflationary solutions with a brief discussion on their main features and a comparison to previous work which can be found in the literature and was mentioned in this introduction. Section V contains a summary of this work.

\section{THE MODEL}

In the KK theory the compactification of the extra dimensions yields a classical unified theory of gravitation and the Yang-Mills fields. The dilaton is defined during the process of dimensional reduction, as a function of the scale factor of internal dimensions. The simplest KK model consists on a five-dimensional space projected into four-dimensions. Then, the unified $U(1)$ Yang-Mills field is associated to electromagnetism and the dilaton may play the role of the scalar field required for inflationary models.

After compactification, Kaluza-Klein and superstring theories are both special cases of a more general Lagrangian given by

$$
\mathcal{L}_{\text {dil }}=\sqrt{-g}\left[(16 \pi G)^{-1} \mathcal{R}-2(\nabla \Phi)^{2}+e^{-2 \alpha \Phi} F_{\mu \nu} F^{\mu \nu}\right]+\mathcal{L}_{\text {matter }}
$$

Lagrangian (II) contains the KK and the SS theories for $\alpha=\sqrt{3}$ and $\alpha=1$ respectively and the Einstein-Maxwell one for $\alpha=0$. After a conformal transformation, the Brans-Dicke theory is also contained here for $\alpha=1 / \sqrt{2 \omega+3}$ (where the parameter $\omega$ is a measure of the influence of the scalar field on the gravitational field). The dilaton field $\Phi$ is coupled to an electromagnetic potential with Faraday tensor given by $F_{\mu \nu}$.

In the attempt to construct a cosmological model that keeps all the possible Universe components, we start from Lagrangian (11) and assume that $\mathcal{L}_{\text {matter }}=\mathcal{L}_{\text {fluid }}-\sqrt{-g}[V(\Phi)+\Lambda]$, 
where $\mathcal{L}_{\text {fluid }}$ represents the content of ordinary matter which is supposed to be a perfect fluid with energy-momentum tensor $T=\operatorname{diag}(-\rho, p, p, p)$, equation of state $p=\omega \rho$ (with $\omega$ constant), and will redshift as $\rho=\rho_{0} a^{-3(1+\omega)}$. We have thus added a model for the fermionic sector to the bosonic part emerging from dilaton theory. We are considering the possibility of a non-vanishing vacuum energy density and the inclusion of $V(\Phi)$ brings the possibility of a phase transition in the early universe. As we said, this is feasible for a KK model, where there are no prescriptions for the potential at low energies. It has been seen that KK, as a fundamental theory, does not lead to successful inflation [12], [21]. Nevertheless, if we take it as a model for a higher-D theory, supplementing it with a Higgs potential, the differential equations of the theory reduce to the standard cosmological equations ((3) below).

In this way, Lagrangian (1) transforms into

$$
\mathcal{L}_{\text {eff }}=\sqrt{-g}\left[\frac{\mathcal{R}}{2}-2(\nabla \Phi)^{2}-V(\Phi)-\Lambda\right]+\mathcal{L}_{\text {fluid }}
$$

where we have taken $8 \pi G=1$ and we have dropped the electromagnetic interaction in order to have isotropy and homogeneity. Therefore the parameter $\alpha$, which gives the three different types of theories, is lost and they are put at the same (mathematical) level for this situation.

For a homogeneous and isotropic space we use the Friedmann-Robertson-Walker metric $d s^{2}=-d t^{2}+a^{2}\left[\frac{d r^{2}}{1-k r^{2}}+r^{2}\left(d \theta^{2}+\sin \theta d \varphi^{2}\right)\right]$. Then, the field equations derived from (因) are

$$
\begin{gathered}
\ddot{\phi}+3 H \dot{\phi}+\frac{\partial V(\phi)}{\partial \phi}=0 \\
\frac{d \rho}{d t}+3 H(\rho+p)=0 \\
3 H^{2}+3 \frac{k}{a^{2}}=\rho+\frac{1}{2} \dot{\phi}^{2}+V(\phi)+\Lambda,
\end{gathered}
$$

where $H=\dot{a} / a$ is the Hubble parameter, a dot means derivation with respect to cosmic time $t$ and we have made the following transformation $\Phi=\frac{1}{2} \phi$.

For a potential of the form $V_{ \pm}(\phi)=\lambda\left(\phi^{2} \pm \delta^{2}\right)^{2}$, we were able to find exact inflationary solutions for this set of equations.

\section{SOLUTIONS WITH $V_{+}(\phi)=\lambda\left(\phi^{2}+\delta^{2}\right)^{2}$}

The scale factor of the universe is found to be 


$$
a(t)=a_{0} \sin ^{2}(\sqrt{\lambda} \delta t)
$$

where $\Lambda=-12 \lambda \delta^{2}, a_{0}=\sqrt{\frac{2 k}{\lambda \delta^{4}}}$ and $\rho_{0}=12 \lambda \delta^{2} a_{0}$. For this solution, we require an inflationary perfect fluid with $w=-2 / 3$, a negative cosmological constant $\Lambda$ and a closed topology of the universe, i.e., $k=+1$.

The scalar field is given by

$$
\phi(t)=\delta \cot (\sqrt{\lambda} \delta t)
$$

The total energy density $\rho_{\text {total }}=\rho+\frac{1}{2} \dot{\phi}^{2}+V_{+}(\phi)+\Lambda$ (fig. 1) is dominated by the scalar field at early times, resulting in an inflationary period from the beginning to $\frac{1}{2} \sqrt{\frac{-\Lambda}{3}} t+\frac{\pi}{2}=\frac{\pi}{4}$; at later times, the influence of the negative cosmological constant closes the universe. In between, the inflationary fluid may, depending on the values of the parameters of the theory, represent the dominant contribution.

It is interesting to notice that, even though the total energy density in the early universe is dominated by the scalar field, the same expansion law would have been obtained in a flat universe, filled just with an inflationary perfect fluid and the (negative) cosmological constant since, with our solution, the full Friedmann equation can in fact be divided into two blocks in which the curvature term is cancelled by the scalar field energy (both potential and kinetic) and the Hubble parameter is equal to the perfect fluid and the $\Lambda$ terms. In fact, the effective cosmological constant is, when the scalar field has settled down, $\Lambda_{e f f}=-12 \lambda \delta^{2}+\lambda \delta^{4}$, which can be positive. Nonetheless, this does not modify the expansion since the positive term $\left(\lambda \delta^{4}\right)$ does not dictate directly the Hubble parameter behaviour.

The evolution of $\phi$ is such that it starts in $+\infty$, goes to its minimum value $(\phi=0)$ when the universe reaches the point of maximum expansion and then to $-\infty$. $\phi$ never settles down in the minimum of its potential but, for small values of the parameters of the theory $-\delta^{2}$ and the self-coupling constant $\lambda$, or equivalenty the value of the cosmological constantit can spend a long time near its vacuum value. This behaviour is shown in fig. 2 .

The universe presents a cyclic behaviour with period $T=4 \pi \sqrt{\frac{-3}{\Lambda}}$, with subsequent deflationary and inflationary episodes. $\phi$ is discontinouos at each point where $a=0$. But, if we identify the $-\infty$ and $+\infty$ values in the potential $V(\phi)$, forming a "compactified potential", then, the universe goes through the "BB" passing by the identified $\pm \infty$ point (see fig.3). Strictly speaking, the set $V_{+}=\left\{(x, y) \in R^{2} \mid y=\left(x^{2}+\delta^{2}\right)^{2}\right\}$ is non-compact, but the set $V_{+}^{C}=V_{+} \cup\{\infty\}$ is, if we use the canonical topology of compactified sets, as it is shown 
in fig.3. The motivation for this compactification comes from the graphs for $V_{+}(\phi)$ and $\phi$, which are in the $R^{2}$ plane. If we compactify $R^{2}$ to $S^{2}$, i.e. if we bend $R^{2}$ untill we obtain a 2-dimensional sphere, the graphs for $V_{+}(\phi)$ and $\phi$ can be extended on the sphere, forming continuous functions.

Notice that for the empty Lemaître models, i.e. non-zero cosmological constant, with positive curvature, only a positive $\Lambda$ is allowed (see e.g. 22]). Here, allowing for other components in the universe, we were able to find an exact solution for a model with positive curvature and negative cosmological constant.

\section{SOLUTIONS WITH $V_{-}(\phi)=\lambda\left(\phi^{2}-\delta^{2}\right)^{2}$}

We have again solutions for an inflationary perfect fluid, with $w=-2 / 3$ and we were able to find a solution only for a universe with positive curvature. The universe experiences an inflationary expansion, as expected, since all the energy sources are inflationary. The inflationary scalar field decays to $\delta$ (see below) but, due to the presence of the non-vanishing cosmological term, the universe never recovers from the inflationary stage. As in the previous case, although the influence of the perfect fluid on the total energy density can be felt only in a reduced epoch (see fig.4), its presence is necessary for the existence of our solution. The evolution of the Hubble parameter depends in fact, as in the previous case, from the $\Lambda$ and the perfect fluid terms only.

The scale factor of the universe is now given by

$$
a(t)=a_{0} \sinh ^{2}(\sqrt{\lambda} \delta t)
$$

with $\Lambda=12 \lambda \delta^{2}, a_{0}=\sqrt{\frac{2 k}{\lambda \delta^{4}}}$ and $\rho_{0}=12 \lambda \delta^{2} a_{0}$. We require $k=+1$ and a positive cosmological constant.

The evolution of the scalar field is

$$
\phi(t)=\delta \operatorname{coth}(\sqrt{\lambda} \delta t)
$$

The scalar field starts in $+\infty$ and quickly settles to $(+\delta)$, without passing through $\phi=0$ (see fig. 5). The potential energy comes then from infinity to its minimum so that, although $V_{-}(\phi)$ is a symmetry breaking potential, since $V_{-}(\phi)$ never takes the value of its local maximum in $V_{-}(\phi=0)$, a phase transition is not achieved through the local maximum. For the case of an oscillating universe, the point $t=0$ could be defined in any point where 
$a=0$. Here, $t=0$ corresponds to the only point where the scale factor goes to zero, defining in such a way negative times for the left branch, where the universe is deflationary. This phase of accelerated contraction preceeding the big bang, corresponds to a time reversal transformation from the $t>0$ solution and can be considered as a particular case of the pre-big-bang scenario [23]. So, if we consider the negative $t$ branch, $\phi$ is settled in one of the true vacuum states $(-\delta)$, then it climbs the potential in the negative $\phi$ value branch to $-\infty$ when $t=0$. After the $\mathrm{BB}, \phi$ rolls down from $+\infty$ to $+\delta$. In such a way, the scalar field goes from $-\delta$ (in the pre-BB) to $+\delta$ (in our universe) through infinity and, if we again suppose that the $-\infty$ and $+\infty$ values are identified in the potential $V_{-}(\phi)$, forming a "compactified potential" $V_{-}^{C}=V_{-}(\phi) \cup\{\infty\}$, the universe achieves an exotic phase transition passing through the identified $\mp \infty$ point (see fig.6).

Our solutions lead, in both cases, to an initial singularity, no matter how high is the value of $\Lambda$. Of course, this approach has limitations: in the vicinity of the Planck scale, the low-energy effective action does not apply and some modifications must be introduced. Then, following the suggestion in [19], taking into account a self-dual dilaton potential could help avoiding the singularity, as in [20].

Another important modification that should be introduced at very early epochs comes from the finite temperature correction terms.

\section{CONCLUSIONS.}

Keeping all the usually accepted possible components of the early universe: the curvature term, a perfect fluid, a scalar field and a cosmological constant term, we found two classes of exact inflationary solutions, with some constraints on the allowed values of the envolved physical quantities. We were able to find exact solutions only for a closed universe with a perfect fluid with equation of state $p=-(2 / 3) \rho$. Then, our models are not realistic in the sense that they do not describe radiation or matter dominated epochs, nevertheless they give the possibility to follow the evolution of a many components universe and verify the fulfillment of the inflationary regime. Our solutions can be a good approximation to the universe behaviour even at later epochs: for instance, a recent work from Turner and White [24] shows that the best fit to all present cosmological data is obtained with a flat CDM universe made of $\Omega_{\text {matter }} \sim 0.3, h \sim 0.7$ and a smooth component contributing the remaining energy density, with equation of state $p_{X}=w \rho_{X}$, with $w \sim-0.6$. Our perfect fluid with 
equation of state $p=-(2 / 3) \rho$, with a specific selection of the set of parameters, could have this role in the present universe.

The influence of the scalar field on the universe evolution basically dominates near the past and future singularity, then it decays and the universe behavior is dictated at late times by the cosmological constant. In order to obtain exact solutions, none of the components in our model could be dropped out. In the class with negative $\Lambda$, the universe has an inflationary epoch with a graceful exit, and then recollapses under the influence of the cosmological constant. This kind of solutions does not allow a symmetry breaking potential. For the class of solutions with a non-vanishing positive cosmological term, the universe enters an inflationary stage but, as expected, never recovers from it. In both cases we have cosmologies with a big bang: even a high cosmological constant value is not able to prevent the shrink to a singularity.

Even in the case where the scalar field has a symmetry breaking potential $V_{-}(\phi)$, its evolution is such that it does not go through a phase transition; at least in this analysis where the finite-temperature effects are not taken into account.

A more complicated and interesting scenario, with several epochs of inflationary and deflationary regimes, arises when considering a pre-big-bang phase, resulting from S-duality. Then, a possible interpretation of the evolution of the scalar field through the $t=0$ point,

rendering it smooth, emerges from invoking a compactified potential at the transition epoch between the two dual phases. This compactification, in the case of the $V_{-}(\phi)$ potential, leads to a non-standard phase transition, that we call exotic phase tarnsition, where the scalar field goes from one minimum to the other passing through the identified $\pm \infty \mathrm{p}$

oint. The initial singularity is somehow contrary to the spirit of a pre-big-bang cosmology, but it has to be stressed that we are using the low-energy effective action near the Planck scale, where in fact we are not expecting it to be valid, so that some modifications must be introduced in order to test the Universe behaviour near " $t=0$ ".

\section{ACKNOWLEDGMENTS}

This work is partially supported by CONACyT-México. MM is grateful to CONACyT for the support through fellowship Reg. No. 91825. GP acknowledges the support from UNAM-DGAPA, IN-109896. 
[1] A. D. Linde. Particle Physics and Inflationary Cosmology. Harwood Academic Publishers, Chur (1990).

[2] A.H. Guth. Phys. Rev. D23, 347 (1981).

[3] F. Lucchin and S. Matarrese, Phys. Rev. D32, 1316 (1985).

[4] S. Kalara, N. Kaloper and K.A. Olive, Nucl. Phys. B341, 252 (1990).

[5] K.A. Olive, Phys. Pep. 190, 307 (1990).

[6] A. D. Linde and A. Mezhlumian, Phys.Rev. D52, 6789 (1995).

[7] L.P. Chimento and A.S. Jakubi, Int. J. Mod. Phys. D5 71 (1996).

[8] F. E. Schunck and E. W.. Mielke, Phys. Rev. D50, 4794 (1994).

[9] E. W. Mielke and F. E. Schunck, Phys. Rev. D52, 672 (1995).

[10] S.M. Carroll and W.H. Press, Annu. Rev. Astron. Astrophys. 30, 499 (1992).

[11] B.A. Campbell, A. Linde, K.A. Olive, Nucl. Phys. B355, 146 (1991).

R. Holman, E.W. Kolb, S.L. Vadas and Y. Wang, Phys. Rev. D43, 995 (1991).

P. Binétruy and M.K. Gaillard, Phys. Rev. D34, 3069 (1986).

[12] J. Levin, Phys. Lett. B343, 69 (1995).

[13] A. Macias, A. Camacho and T. Matos, Int. J. Mod. Phys. D5 617 (1995).

[14] Y M Cho, J.Math.Phys., 16, 2029 (1975).

[15] R. Brustein and P.J. Steinhardt, Phys. Lett. B302, 196 (1993).

[16] A. Font, L.E. Ibáñez, D. Lüst and F. Quevedo, Phys. Lett. B249, 35 (1990).

[17] A. De la Macorra, S. Lola, Phys. Lett. B373, 299 (1996).

[18] E.J. Copeland, A.R. Liddle, D. H. Lyth, E. D. Stewart and D. Wands, Phys. Rev. D49, 6410 (1994).

[19] M.Gasperini and G. Veneziano, Astropart. Phys. 1317 (1993).

[20] C. Angelantonj, L. Amendola, M. Litterio and F. Occhionero, Phys. Rev. D 511607 (1995).

[21] A. Camacho, Master Thesis, UAM-IZtapalapa (1994).

[22] H. C. Ohanian and R. Ruffini, Gravitation and Spacetime, 2nd ed. W.W. Norton E company (1994).

[23] M. Gasperini, The inflationary role of the dilaton in string cosmology, Proceedings of the international workshop "Birth of the Universe and Fundamental Physics", Rome, 18-21 May 1994, ed. F. Occhionero, Springer-Verlag, Berlin (1995).

[24] M. Turner and M. White, Phys. Rev. D56 4439 (1997). 


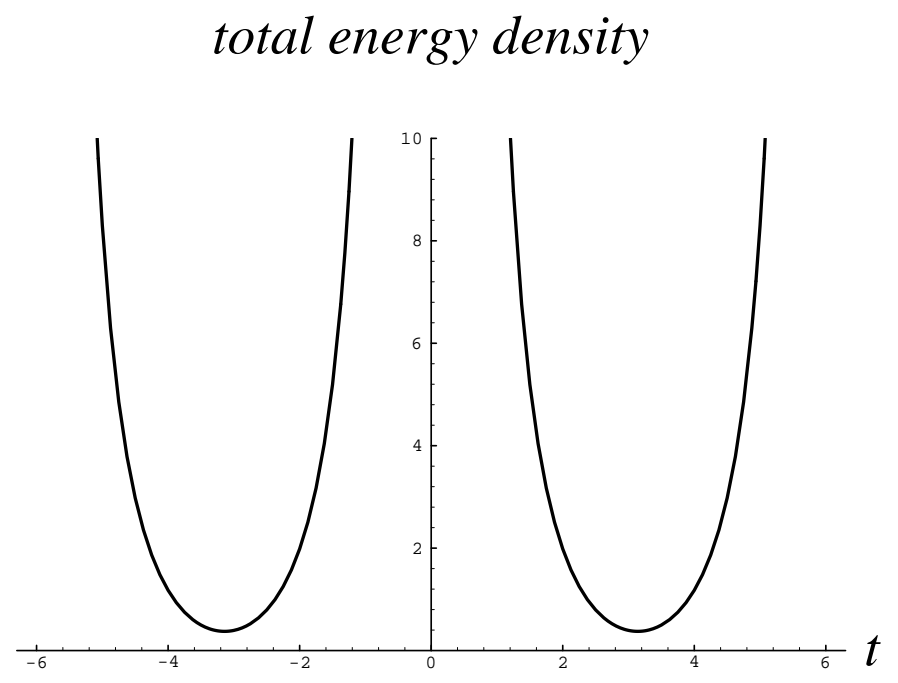

FIG. 1. The total energy density $\rho_{\text {total }}=\rho+\frac{1}{2} \dot{\phi}^{2}+\lambda\left(\phi^{2}+\delta^{2}\right)^{2}+\Lambda$ for the case $w=-\frac{2}{3}$, negative $\Lambda$ and $k=+1$, with $\lambda=+\frac{1}{4}$ and $\delta=1$. 
scalar field

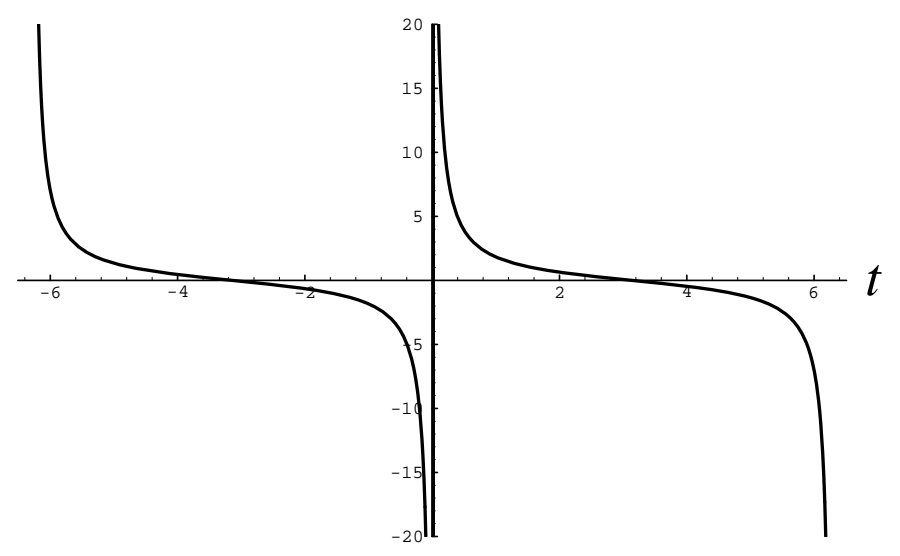

FIG. 2. The scalar field $\phi$ associated to $V_{+}(\phi)$, with $k=+1, \lambda=\frac{1}{4}$ and $\delta=1$. 


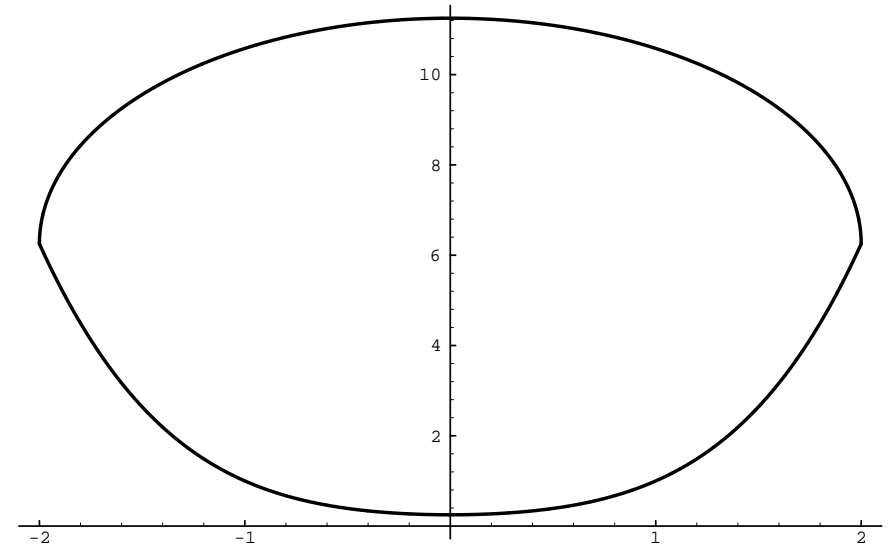

FIG. 3. The compactified potential field $V_{+}^{C}=V_{+}(\phi) \cup\{\infty\}=\lambda\left(\phi^{2}+\delta^{2}\right)^{2} \cup\{\infty\}$, where we have identified $-\infty$ and $+\infty$ at the $t=0$ point. 


\section{total energy density}

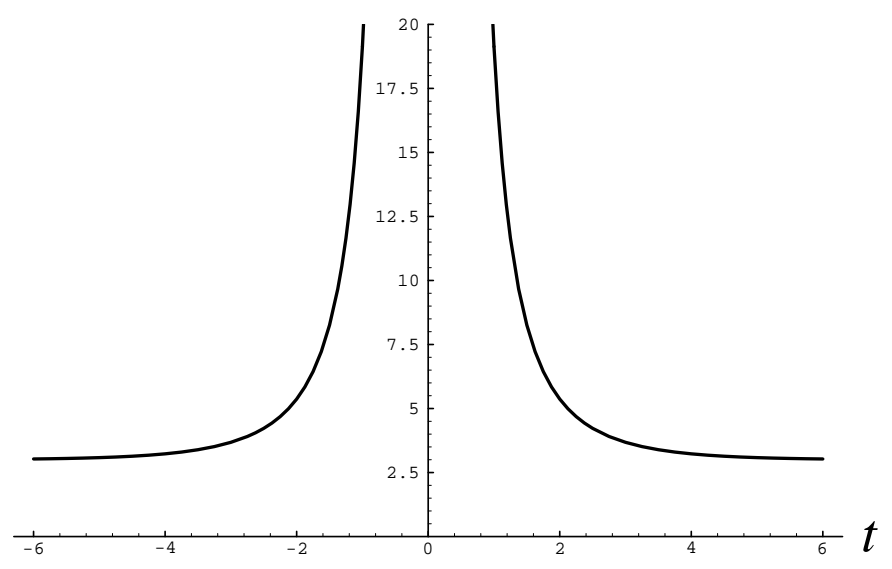

FIG. 4. The total energy density $\rho_{\text {total }}=\rho+\frac{1}{2} \dot{\phi}^{2}+\lambda\left(\phi^{2}-\delta^{2}\right)^{2}+\Lambda$ for the case $w=-\frac{2}{3}$, positive $\Lambda$ and $k=+1$, with $\lambda=\frac{1}{4}$ and $\delta=1$. 
scalar field

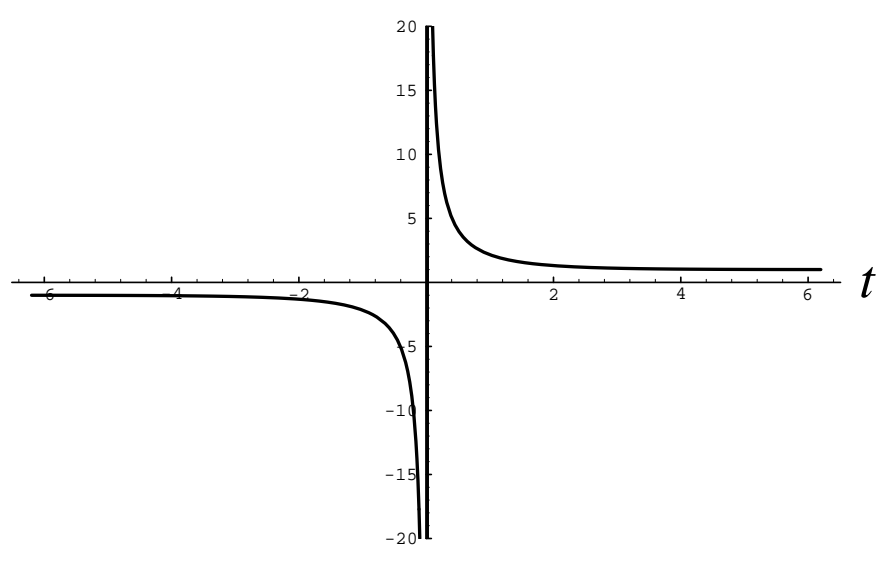

FIG. 5. The scalar field $\phi$ associated to $V_{-}(\phi)$, with $k=+1, \lambda=\frac{1}{4}$ and $\delta=1$. 


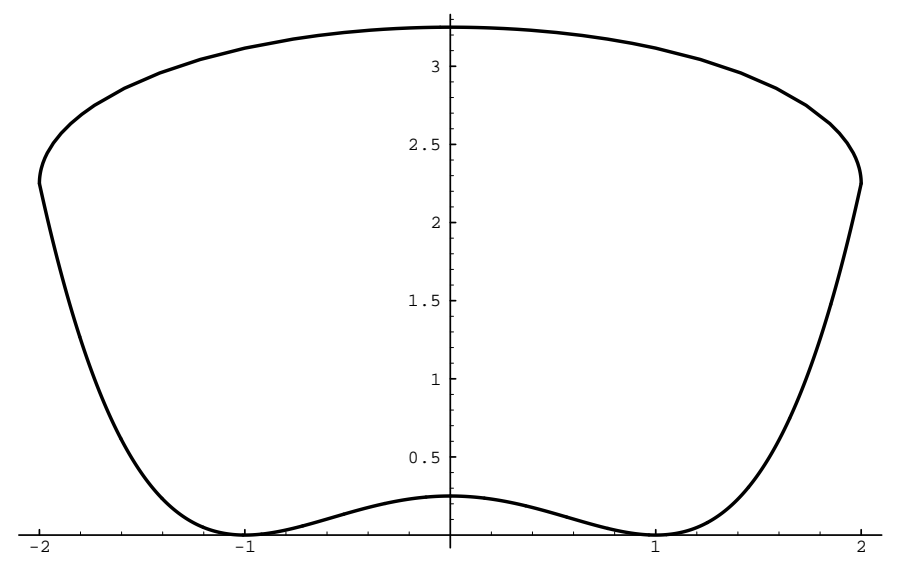

FIG. 6. The compactified potential field $V_{-}^{C}=V_{-}(\phi) \cup\{\infty\}=\lambda\left(\phi^{2}-\delta^{2}\right)^{2} \cup\{\infty\}$, where we have identified $-\infty$ and $+\infty$ at the $t=0$ point. 